\title{
Dienstleistung für die MST-Ausbildung in Thüringen
}

Naue, Dietmar

BWAW Bildungswerk für berufsbezogene Aus- und Weiterbildung Thüringen gGmbH

Peter-Cornelius-Str. 12, 99096 Erfurt

\section{Die Mikrosystemtechnik in Thüringen}

3 der 20 durch IVAM Research identifizierten Mikrotechnik-Cluster in Deutschland befinden sich in Thüringen. Ihnen gehören 60 (18\%) der 322 kleinen und mittleren Unternehmen aus deutschen Mikrotechnik-Clustern an. ${ }^{1}$ Die Unternehmenslandschaft ist dabei vor allem von Unternehmen mit unter 50 Beschäftigten geprägt.

Wie Abbildung 1 anhand ausgewählter in Thüringer Unternehmen und Einrichtungen relevanter Gebiete der Mikro- und Nanotechnologien, darunter der Mikrosystemtechnik (MST), zeigt, ist eine große Vielfalt an Produkten, Technologien, Verfahren, und Werkstoffen charakteristisch. Immer mehr Unternehmen verfolgen dabei die Strategie, sich verstärkt auch der Systemintegration ihrer Komponenten zuzuwenden.

\begin{tabular}{|c|c|c|}
\hline Technologie/Verfahren & Produkte & Dienstleistungen \\
\hline $\begin{array}{l}\text { Mikroelektronik, Optoelektronik } \\
\text { - Dotierverfahren } \\
\text { - CVD, PVD }\end{array}$ & $\begin{array}{l}\text { Elektronische Komponenten } \\
\text { - ASICs } \\
\text { - Bestückte Leiterplatten } \\
\text { - RFID-Baugruppen }\end{array}$ & $\begin{array}{l}\text { Electronic } \\
\text { Manufacturing } \\
\text { Services }\end{array}$ \\
\hline $\begin{array}{l}\text { Mikromechanik } \\
\text { - Si-Tiefätzen } \\
\text { - Laserbearbeitung } \\
\text { - Ultrapräzisionsbearbeitung }\end{array}$ & $\begin{array}{l}\text {-RFID-Baugruppen } \\
\text { Mikrosensoren } \\
\text { - Beschleunigungssensoren } \\
\text { - Gassensoren } \\
\text { - Drucksensoren }\end{array}$ & \multirow[t]{7}{*}{ IC-Entwurf } \\
\hline $\begin{array}{l}\text { Mikrooptik } \\
\text { - Heißprägen }\end{array}$ & $\begin{array}{l}\text { Solarwafer/-zellen/-module } \\
\text { - Kristalline Wafer/Zellen/Module }\end{array}$ & \\
\hline \multirow{3}{*}{$\begin{array}{l}\text { Aufbau-/Verbindungstechnik } \\
\text { - Wafer-, Chip-, Drahtbonden } \\
\text { - SMT } \\
\text { - LTCC-Technik }\end{array}$} & - Dünnschichtmodule & \\
\hline & $\begin{array}{l}\text { Mikroaktoren } \\
\text { - Piezoaktoren }\end{array}$ & \\
\hline & RFID-Systeme & \\
\hline Systemintegration & Nanokomposite & \\
\hline $\begin{array}{l}\text { Chemische/physikalische } \\
\text { Nanotechnologie } \\
\text { - Sol-Gel-Verfahren } \\
\text { - Mahlen }\end{array}$ & $\begin{array}{l}\text { Nanobeschichtete/-strukturierte } \\
\text { Komponenten } \\
\quad \text { - Nanobeschichtete Linsen }\end{array}$ & \\
\hline
\end{tabular}

Abbildung 1: In Thüringer Unternehmen und Einrichtungen relevante Gebiete der Mikro- und Nanotechnologien (Beispiele)

\section{Ausbildung für die Mikrosystemtechnik in Thüringen}

Im Kontext der Ausbildung wird hier bei der Betrachtung der Mikrosystemtechnik auch die Aufbau- und Verbindungstechnik einbezogen, selbst wenn sie nicht in jedem Einsatzfall zu einem Mikrosystem führt. Die relevanten Ausbildungsberufe sind insbesondere der Mikrotechnologe ${ }^{2}$, der Elektroniker für Geräte und Systeme und der Mechatroniker. Von besonderer Bedeutung ist der 1998 geschaffene duale Ausbildungsberuf Mikrotechnologe, der als prozessorientierter, naturwissenschaftlich-technisch basierter Beruf eine große Breite von Tätigkeitsprofilen in den Mikro- und Nanotechnologien, darunter bei der Herstellung von Komponenten für die Mikrosystemtechnik, abdeckt. Die Ausbildung erfolgt entsprechend der Ausbildungsordnung in einem Teil der praktischen Ausbildung in den Schwerpunkten Halbleitertechnik oder Mikrosystemtechnik. Insgesamt werden gegenwärtig in Thüringen 132 Mikrotechnologen in 28 Unternehmen ausgebildet, hinzu kommen 26 Auszubildende in überbetrieblicher Ausbildung. Das sind über $20 \%$ der auszubildenden Mikrotechnologen in der Bundesrepublik. Davon absolvieren 48 Auszubildende in 20 Unternehmen eine Ausbildung im Schwerpunkt Mikrosystemtechnik. 


\section{Gründe und Ansatz für Dienstleistungen zur MST-Ausbildung in Thüringen}

Die betriebliche Ausbildung im Beruf Mikrotechnologe mit der im Rahmenausbildungsplan der Ausbildungsordnung vorgegebenen Komplexität kann von den einzelnen Ausbildungsbetrieben häufig nicht vollständig abgedeckt werden. Zum einen steht den breit angelegten inhaltlichen Ausbildungserfordernissen der hohe Spezialisierungsgrad vieler Unternehmen entgegen. Zum anderen benötigen das geforderte selbständige Planen, Durchführen und Dokumentieren von Arbeitsvorgängen und von Prozessabläufen, das Konzipieren von Tests, Erfassen von Messdaten, ihre statistische Bearbeitung und Auswertung Raum für das „Durchspielen von Varianten“, für das Vermitteln von „AhaEffekten“. Die sensiblen Produktionsprozesse ermöglichen solche Eingriffe jedoch nicht.

Die Unternehmen erwarten zudem im Zusammenhang mit (zukünftigen) Innovationsprozessen, dass die Auszubildenden grundlegende technisch-technologische Entwicklungen bereits kennen lernen, auch wenn sie noch nicht breit Einzug in die Produktion gehalten haben, und sich ausgeprägte Lernkompetenzen aneignen.

In Hochtechnologiebereichen wie der MST können nur spezialisierte Bildungsdienstleister die für die ergänzende Ausbildung spezifischen materiell-technischen und personellen Kapazitäten vorhalten. An einigen Standorten in Deutschland (z. B. Regensburg, Dresden) sind diese Dienstleister eine Struktureinheit großer Unternehmen und übernehmen Ausbildungssequenzen auch für andere Unternehmen der Region. In Thüringen wurde aufgrund des weitgehenden Fehlens relevanter Großunternehmen auf Initiative von Industrieunternehmen, einer wirtschaftsnahen Forschungseinrichtung und dem BWAW mit Unterstützung des Freistaates Thüringen und der IHK der Weg gewählt, ein Kompetenzzentrum für die Aus- und Weiterbildung in der Mikrotechnologie bei einem eigenständigen privatwirtschaftlich agierenden Bildungsdienstleister aufzubauen. Kernstück sind dabei ein Ausbildungsreinraum, ein Labor für Aufbau- und Verbindungstechnik sowie Chemie- und Elektroniklabore. Die Lehrkräfte verfügen über langjährige berufspädagogische Erfahrungen und kommen vielfach aus der Mikroelektronikindustrie.

Die duale Ausbildung der Mikrotechnologen erfolgt deshalb an 3 Lernorten (siehe Abbildung 2).

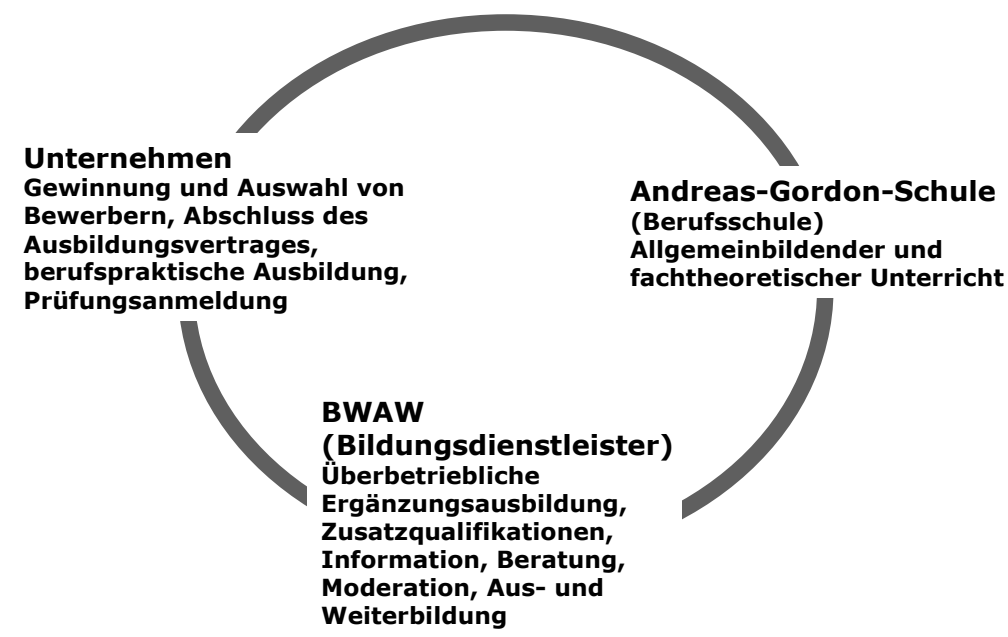

Abbildung 2: Lernorte der Verbundausbildung für Mikrotechnologen in Thüringen

\section{Angebotene Dienstleistungen für die MST-Ausbildung}

Überbetriebliche Ergänzungsausbildung und Vermittlung von Zusatzqualifikationen

Etabliert wurden eine überbetriebliche Ergänzungsausbildung und die Vermittlung von Zusatzqualifikationen, deren Konzept jährlich zwischen dem BWAW, den Unternehmen und der Berufsschule abgestimmt und der IHK vorgelegt wird (vgl. Abbildung 3). Diese angebotene Dienstleistung wird gegenwärtig mit allen Modulen von den ausbildenden Unternehmen in Anspruch genommen und vom Freistaat Thüringen aus Mitteln des Europäischen Sozialfonds (ESF) gefördert. 


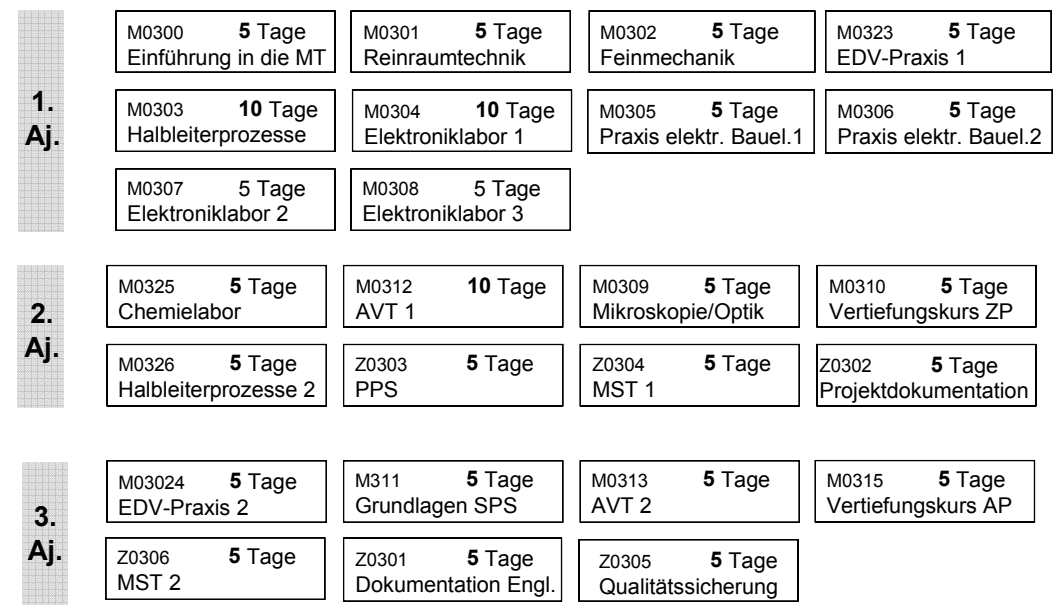

Abbildung 3: Module der überbetrieblichen Ergänzungsausbildung (M) und Vermittlung von Zusatzqualifikationen (Z) im Ausbildungsjahr 2008/2009

Als von besonderer Bedeutung für die Sicherung des ingenieurtechnischen Nachwuchses in Unternehmen hat sich das duale Studium - eine Kombination aus Ingenieurstudium an der FH Schmalkalden bzw. TU IImenau und Ausbildung zum Mikrotechnologen - erwiesen. Neben den Lernorten Hochschule und Unternehmen übernimmt das BWAW hier ebenfalls die ergänzende berufspraktische Ausbildung und vermittelt diejenigen berufstheoretischen Inhalte, die nicht bzW. nicht bis zum Zeitpunkt der IHK-Abschlussprüfung als Mikrotechnologe Bestandteil des Studiums sind. Dieser Weg der Nachwuchssicherung wird gegenwärtig von 8 Mikrotechnologieunternehmen für 48 Auszubildende/Studenten genutzt. Dass darunter bisher erst 3 MST-Unternehmen mit 6 Teilnehmern sind, zeigt das noch ungenutzte Potenzial auf - vor allem unter dem Aspekt, dass es mit den derzeit und in den folgenden Jahren drastisch sinkenden Schulabgängerzahlen in Thüringen bedeutend schwieriger wird, Absolventen ingenieurtechnischer Studienrichtungen für die in der Region ansässigen Unternehmen zu gewinnen.

Unterstützung des Matchings zwischen Unternehmen und Jugendlichen

Mit dem wirtschaftsgetragenen Stufenmodell für die Ausbildung von Mikrotechnologen wurde im JOBSTARTER-Vorhaben EwiS ${ }^{3}$ durch das BWAW gemeinsam mit Mikrotechnologieunternehmen ein Modell entwickelt, das die Einstiegsbarrieren für die Aufnahme einer Ausbildung im Hochtechnologiebereich bei Unternehmen und Jugendlichen deutlich verringert. ${ }^{4}$ Das Modell und die im Zusammenhang damit vom BWAW erbrachten Informations- und Unterstützungsleistungen führten dazu, dass 7 weitere Unternehmen der MST für eine Ausbildung von Mikrotechnologen (Beginn 2007) gewonnen werden konnten.

Netzwerkaufbau, -entwicklung und -moderation

Als sehr positiv bewertet wird von den Unternehmen, dass der vom BWAW verfolgte ganzheitliche Ansatz zur Aus- und Weiterbildung auf dem Gebiet der Mikro- und Nanotechnologien neben den Aktivitäten zur Berufsorientierung, Lehrerfortbildung, Erstausbildung, Vorbereitung neuer Mitarbeiter für Unternehmen sowie berufsbegleitenden Weiterbildung auch Modellvorhaben und Projekte einschließt, die insbesondere

- der Analyse von Bildungsbedarfen in Unternehmen und Einrichtungen,

- der Entwicklung und Erprobung von Qualifizierungsmodellen und Bildungskonzepten sowie

- der Etablierung und Ausgestaltung von Aus- und Weiterbildungsnetzwerken

dienen.

Ausgangspunkt war ein BIBB-Wirtschaftsmodellversuch zur Schaffung eines ganzheitlichen Modells der beruflichen Bildung in der Mikrotechnologie durch einen Bildungsdienstleister im Netzwerk mit Unternehmen, bei dem die Mikrotechnologenausbildung im Mittelpunkt stand. ${ }^{5}$ Beginnend im Jahr 2002 haben dann Unternehmen, eine Universität, der Bildungsdienstleister BWAW, Forschungseinrichtungen und Vereine ein Netzwerk zur „Fachkräftesicherung in der Mikrosystemtechnik in Thüringen (FasiMiT)“ aufge- 
baut. ${ }^{6}$ Eines der forcierten Kompetenzfelder des Netzwerks war und ist dabei die Erstausbildung, insbesondere im Beruf Mikrotechnologe.

Gespräche mit Geschäftsführern und anderen Führungskräften in ca. 70 Unternehmen zur Aus- und Weiterbildung in der MST und verschiedene Veranstaltungen der MST-Community wurden in diesem und im Projekt EwiS (s. o.) dazu genutzt, das Berufsbild vorzustellen und die Ausbildung unterstützende Aktivitäten aufzuzeigen.

Berufs- und disziplinenübergreifend in Zusammenarbeit mit Unternehmen den Bildungsbedarf (einschließlich Nachwuchsgewinnung) zu erkennen, entsprechende Bildungsdienstleistungen für verschiedene Zielgruppen vorzuhalten und zu realisieren, bleibt der Anspruch des Netzwerks FasiMiT und seiner Koordinierungsstelle beim BWAW auch nach der ausgelaufenen BMBF-Förderung. Flankiert wird diese Arbeit durch eine enge Verbindung zu relevanten Clusterinitiativen in Thüringen (insbesondere dem MNT Mikro-Nanotechnologie-Thüringen e. V.) und den darin mitwirkenden Unternehmen sowie zu weiteren Akteuren bundesweit.

Aufbauend auf Ergebnissen eines BIBB-Wirtschaftsmodellversuchs hat das Netzwerk FasiMiT das „Forum der Mikrotechnologenausbildung in Thüringen“ mit jährlich 1-2 Treffen von Akteuren der unterschiedlichen Lernorte und weiteren Partnern als ständige Plattform des Erfahrungsaustausches, der Abstimmung zur Lernortkooperation und für Diskussionen zur Weiterentwicklung der Ausbildung fest etabliert. Moderiert wird das Forum durch das BWAW. Eine solche Plattform ist für die Ausbildung für die MST unter dem Aspekt von besonderer Bedeutung, dass bei der MST im Unterschied zur Halbleitertechnik im Wesentlichen nicht die Technologieanbieter (die Hersteller von Mikrosystemen und deren Komponenten), sondern die Kunden und die Endnutzer die Roadmap technisch-technologischer Entwicklung bestimmen. ${ }^{7}$ Dies erschwert die Erkennung langfristiger Trends für Anforderungen an Bildung. Bewährt hat sich deshalb, möglichst umfassend die Expertise aller an der Ausbildung beteiligten Akteure zu nutzen.

\section{Überregionaler Transfer der Erfahrungen}

FasiMiT hat seine Erkenntnisse und Erfahrungen in der Mikrotechnologenausbildung in das von ihm koordinierte Themenfeld "Gewerbliche Ausbildung Mikrotechnologen/-innen, Neuordnung, Aufstiegsqualifizierung" der 6 vom BMBF geförderten MST-Aus- und Weiterbildungsnetzwerke (AWNET) eingebracht und gleichzeitig von der Arbeit des Themenfeldes profitiert. Ausgehend von der Diskussion der Ergebnisse einer deutschlandweiten Befragung von Experten der Mikrotechnologenausbildung in einem Workshop im September 2008 erarbeitet gegenwärtig eine überregionale Arbeitsgruppe unter Federführung des BWAW eine Handreichung für die Mikrotechnologenausbildung, in die auch die Thüringer Erfahrungen mit Dienstleistungen für die MST-Ausbildung eingehen werden.

\section{Ausblick}

Die überbetriebliche Ergänzungsausbildung und Vermittlung von Zusatzqualifikationen für Mikrotechnologen und andere Berufe werden entsprechend den spezifischen Anforderungen und Bedingungen der Unternehmen der Halbleitertechnik, Mikrosystemtechnik, Aufbau- und Verbindungstechnik sowie Photovoltaikindustrie weiter profiliert. Dabei berücksichtigt werden auch Schnittstellen zu anderen Hochtechnologien, insbesondere zur Nanotechnologie, Biotechnologie und den Optischen Technologien.

Eng damit verbunden ist, dass mit Unterstützung des Freistaates Thüringen in bewährter Public-PrivatePartnership ein Thüringer Kompetenzzentrum für Hochtechnologien und Solarwirtschaft beim BWAW errichtet wird. Damit wird die materiell-technische Basis für Dienstleistungen zur Ausbildung in Hochtechnologien bedeutend verbessert.

Das BWAW wird sich gemeinsam mit seinen Partnern in Überlegungen zur Weiterentwicklung von Berufen und Berufsgruppen sowie zur Gestaltung der Ausbildung einbringen. 
Mit der auch in Thüringen geplanten Umsetzung des Weiterbildungsmodells Spezialisten/ Prozessmanager-Mikrotechnologie auf der Grundlage der bundesweit gültigen Fortbildungsordnung werden die Möglichkeiten der beruflichen Karriereentwicklung für Mikrotechnologen und andere Facharbeiter wesentlich erweitert.

Kontakt:

d.naue@bwaw-thueringen.de

\footnotetext{
${ }^{1}$ MST-Atlas Deutschland 2005. Mikrosystemtechnik-Cluster in Deutschland. Hrsg.: IVAM Research, Dortmund 2005, http://www.ivam-research.de/de/index.php?content=studien_details\&id=1 vom 10.02.09.

${ }^{2}$ Bei allen Berufs- und Funktionsbezeichnungen in diesem Dokument sind die männliche und weibliche Form gleichermaßen gemeint.

3 JOBSTARTER-Vorhaben „Entwicklung und Erprobung eines wirtschaftsgetragenen Stufenmodells für den Ausbildungsberuf Mikrotechnologe/-in (EwiS)“, gefördert aus Mitteln des Bundesministeriums für Bildung und Forschung und der Europäischen Union - Europäischer Sozialfonds, Laufzeit: 2006 - 2008.

${ }^{4}$ Siehe: Übergänge von der Schule in die Berufsausbildung erfolgreich gestalten - Erfahrungen und Erkenntnisse aus der Entwicklung und Erprobung eines wirtschaftsgetragenen Stufenmodells für den Ausbildungsberuf Mikrotechnologe/Mikrotechnologin. Ratgeber für Ausbildungsunternehmen und Bildungsdienstleister. Hrsg.: BWAW, Dezember 2007.

${ }^{5}$ BIBB-Wirtschaftsmodellversuch „Moderne Aus- und Weiterbildung im Hochtechnologiebereich Mikrotechnologie durch Bildungsdienstleister im Netzwerk von Unternehmen (MikroTec)“, gefördert durch das Bundesministerium für Bildung und Forschung und das Thüringer Ministerium für Wirtschaft, Technologie und Arbeit aus Mitteln des Europäischen Sozialfonds, Laufzeit: $2001-2005$.

${ }^{6}$ Projekt „Aus- und Weiterbildungsnetzwerk zur Fachkräftesicherung in der Mikrosystemtechnik in Thüringen (Fasi$\mathrm{MiT})^{\prime}$, gefördert durch das Bundesministerium für Bildung und Forschung, Laufzeit: 2002 - 2008. Siehe auch die Broschüre „Aus- und Weiterbildungsnetzwerk zur Fachkräftesicherung in der Mikrosystemtechnik in Thüringen. Partner für Unternehmen in den Mikro- und Nanotechnologien“. Hrsg.: FasiMiT, vertreten durch BWAW, November 2007, sowie die Homepage www.fasimit.de.

${ }^{7}$ Siehe Salomon, van Heeren: Die Zulieferkette der Mikrosystemtechnik: Dienstleistungen und Infrastruktur. In: inno, Nr. 29, März 2005.
} 